\title{
Hormonal networks involved in apical hook development in darkness and their response to light
}

\author{
Maria A. Mazzella ${ }^{1 *}$, Jorge J. Casal ${ }^{2,3}$, Jorge P. Muschietti ${ }^{1,4}$ and Ana R. Fox ${ }^{1+}$ \\ 1 Instituto de Investigaciones en Ingeniería Genética y Biología Molecular, "Dr. Héctor Torres" (INGEBI-CONICET), Buenos Aires, Argentina \\ ${ }^{2}$ Facultad de Agronomía, Instituto de Investigaciones Fisiológicas y Ecológicas Vinculadas a la Agricultura, Universidad de Buenos Aires and CONICET, Buenos \\ Aires, Argentina \\ ${ }^{3}$ Fundación Instituto Leloir, Instituto de Investigaciones Bioquímicas de Buenos Aires-CONICET, Buenos Aires, Argentina \\ ${ }^{4}$ Departamento de Biodiversidad y Biología Experimental, Facultad de Ciencias Exactas y Naturales, Universidad de Buenos Aires, Buenos Aires, Argentina
}

\section{Edited by:}

Tohru Hashimoto, Uozaki Life

Science Laboratory, Japan

\section{Reviewed by:}

Teva Vernoux, Centre National de la Recherche Scientifique, France Nobuahru Fujii, Tohoku University, Japan

Xing Liu, University of Minnesota, USA

\section{*Correspondence:}

Maria A. Mazzella, INGEBI, Instituto de Investigaciones en Ingeniería

Genética y Biología Molecular, "Dr. Héctor Torres," 2490 Vuelta de

Obligado, Buenos Aires, 1428, Argentina

e-mail:mazzella@dna.uba.ar

${ }^{\dagger}$ Present address:

Ana R. Fox, Instituto de Genética

Ewald A. Favret (CICVYA-INTA),

Buenos Aires, Argentina

\section{INTRODUCTION}

Endogenous signals such as hormones control the developmental progress during the life cycle of plants. However, as sessile organisms, plants have evolved the ability to dynamically adjust their body form and function in response to the changing environment. These changes tailor the plant phenotype to the prevailing conditions, thus favoring plant survival (Casal et al., 2004). Light is one of the most influential external stimuli controlling plant development. For instance, when seeds germinate buried in the darkness of the soil, the new seedlings follow the skotomorphogenic pattern. In Eudicots, this developmental program is characterized by a fast growing embryonic stem (e.g., hypocotyl in Arabidopsis thaliana), the formation of an apical hook structure and the presence of folded cotyledons. Light exposure initiates the transition between skoto- to photomorphogenesis (Kami et al., 2010). The hypocotyl reduces its growth rate, the apical hook opens and the cotyledons unfold while the seedling becomes photosynthetically competent.

In this paper we review the interplay between light signals perceived by photoreceptors (Box 1) and hormonal signals in the control of apical hook development and opening. The main focus is placed on Arabidopsis thaliana but information from other species is included to provide a more complete picture.

\section{THE FUNCTION OF THE APICAL HOOK}

The apical hook is a transient structure that results from the bending in approximately $180^{\circ}$ of the hypocotyl at its apex, below the cotyledons [wild type (WT) in Figure 1]. The apical hook of dark-grown seedlings develops after seed germination to minimize the damage to the shoot apical meristem in its way through the soil to reach the light. The opening of this transient structure formed in the dark must be tightly regulated to ensure seedling survival: if the hook opens prematurely before emergence from the soil, the cotyledons and meristem could be damaged; if the hook opens late, seed reserves could be consumed before the cotyledons become fully exposed to light for photosynthesis.

\section{CELL GROWTH DURING APICAL HOOK FORMATION, MAINTENANCE AND OPENING}

Apical hook development takes place in three consecutive phases: formation, maintenance and opening (Raz and Ecker, 1999) (Figure 1). In A. thaliana seedlings grown in darkness, the formation phase lasts approximately $26 \mathrm{~h}$ after germination while the maintenance phase, where the hypocotyl remains closed, lasts another $25 \mathrm{~h}$ (Vandenbussche et al., 2010; Zadnikova et al., 2010; Gallego-Bartolomé et al., 2011). Under prolonged darkness, opening will anyway occur $90-120 \mathrm{~h}$ after germination (Vandenbussche et al., 2010; Zadnikova et al., 2010; GallegoBartolomé et al., 2011).

The apical hook is formed as the result of localized cell division and asymmetric cell growth at opposite sides of the apical portion of the hypocotyl. To analyze the contribution of cell division and cell growth, it is useful to consider four sections of the apical hook as defined by the apical and basal sides (along the hypocotyl) 


\section{Box 1 | Light perception and signaling in photomorphogenesis \\ PHOTORECEPTORS}

When the shoot emerges from the soil, the light signal that initiates the transition between skoto- and photo-morphogenesis (de-etiolation) is perceived mainly (although no exclusively) by phyA, phyB and cry1. phyA is important for the early steps of this transition, which is completed by phyA itself under dense canopies and by phyB and cry1 in open places (Sellaro et al., 2010; Casal et al., 2013). phyA is activated by radiation between 300 and $780 \mathrm{~nm}$ range (Shinomura et al., 1996), but maximally by far-red light (Rausenberger et al., 2011). phyB and cry1 are activated by red and blue light, respectively (Quail et al., 1995; Cashmore et al., 1999) (Figure 4).

\section{TRANSCRIPTION FACTORS WITH EITHER POSITIVE OR NEGATIVE EFFECTS ON PHOTOMORPHOGENESIS}

PIFs are bHLH transcription factors that bind mainly to the G-box motifs of their target promoters to repress photomorphogenesis (De Lucas et al., 2008; Leivar et al., 2009; Shin et al., 2009; Zhang et al., 2013b). Conversely, HY5 is a b-Zip transcription factor that binds mainly to the G-box motifs of their target promoters to promote photomorphogenesis (Lee et al., 2007; Zhang et al., 2013a). In some cases, PIFs and HY5 may actually compete for the same binding sites (Chen et al., 2013). Light reduces the activity of PIFs and enhances the activity of HY5 (and many other transcription factors with positive action in de-etiolation) to promote photomorphogenesis (Figure 4).

\section{SIGNAL TRANSDUCTION}

In darkness, phyA and phyB are cytoplasmic homodimers. Light absorption shifts phyA and phyB from the inactive $(\mathrm{Pr})$ to the active (Pfr) form and part of these Pfr pools migrate to the nucleus (Kircher et al., 1999, 2002; Huq et al., 2003), where they bind PIFs (De Lucas et al., 2008; Feng et al., 2008). As a result of this interaction PIFs become phosphorylated and their activity is reduced by a combination of ubiquitination followed by degradation in the 26S proteasome (Al-Sady et al., 2006; Shen et al., 2007) and reduced ability to bind their targets (Park et al., 2012) (Figure 4). cry1 is present in the nucleus and the cytoplasm and light does not significantly change its localization (Wu and Spalding, 2007). In darkness, the E3 ubiquitin-protein ligase COP1 forms a complex with SPA1 and the CUL4-DDB1 E3 ligase core (Lau and Deng, 2012). The multimeric CUL4-DDB1-COP1-SPA1 complex binds ubiquitin to HY5 (and to other transcription factors that promote photomorphogenesis), which becomes targeted to degradation in the $26 \mathrm{~S}$ proteasome. In the light, the active nuclear pools of cry1, phyA and phyB interact with COP1 (Wang et al., 2001; Yi and Deng, 2005; Liu et al., 2011) and reduce COP1-dependent degradation of transcription factors by a combination of disaggregation of the COP1-SPA1 complex (demonstrated for cry1, Lian et al., 2011; Liu et al., 2011) and translocation of COP1 to the cytosol (von Arnim and Deng, 1994; Osterlund et al., 1999). The traslocation of COP1 to the cytoplasm is a fast process that regulates COP1 activity (Pacín et al., 2014), allowing the pool of nuclear HY5 to build up (Osterlund et al., 2000; Pacín et al., 2014).

\section{HORMONES}

The skotomorphogenic pattern requires brassinosteroids (Chory et al., 1991; Li et al., 1996) and gibberellins (Alabadí et al., 2004). Light reduces the levels of gibberellins (Ait-Ali et al., 1999; Achard et al., 2007; Alabadí et al., 2008) by acting on the expression genes involved in their metabolism (O'Neill et al., 2000; Reid et al., 2002; Zhao et al., 2007). In pea this control involves the COP1/HY5 (Weller et al., 2009). The reduction in gibberellins lowers the activity of the GID1 receptor, involved in a complex that targets DELLA to degradation by the ubiquitin-26S proteosome pathway (Ariizumi et al., 2008) (Figure 4). Thus, in the light DELLAs increase their abundance (Achard et al., 2007) and bind PIFs further reducing their activity (De Lucas et al., 2008; Feng et al., 2008). DELLAs also bind to BRASSINAZOLE RESISTANT1 (BZR1), a transcription factor activated by brassinosteroids, and reduce their activity (Bai et al., 2012; Gallego-Bartolome et al., 2012). These changes in hormone signaling reinforce photomorphogenesis.

and the concave (inner) and convex (outer) sides across the hook (Raz and Koornneef, 2001) (Figure 2). When the growth rate of the cells is slower at the inner than at the outer sides of the hypocotyl, the hook is formed and maintained. Conversely, when growth rates at the inner side of the hook exceed those of the outer cells, the hypocotyl straightens and opens (Raz and Ecker, 1999; Vandenbussche and Van Der Straeten, 2004; Vriezen et al., 2004). The main contribution of cell division is evident during the first $24 \mathrm{~h}$ of hook development, when more sub-epidermal cells originate at the apical than at the basal portions of the hook (Raz and Koornneef, 2001) (Figure 2). Slightly more cells can be observed at the outer side than at the inner side (Raz and Koornneef, 2001). In sunflower, cell division occurs in the apical hook in coincidence with the high metabolic activity found in that part of the hypocotyl (Kutschera and Niklas, 2013).

\section{AUXIN GRADIENTS DURING HOOK DEVELOPMENT}

The mechanism underlying differential cell growth in the establishment of hook structure involves a local accumulation auxin at the inner side of the hook (Figure 2). During hook formation and maintenance, the expression of the auxin signaling reporter DR5:GUS localizes to the cortex and epidermal cells of the inner side of the hook (Zadnikova et al., 2010; Gallego-Bartolomé et al., 2011). GUS activity driven by DR5 at the inner side becomes diffuse and fades away during the opening phase. In seedlings treated with an auxin transport inhibitor, no auxin gradient is established, asymmetrical GUS accumulation is blocked and the hook is not formed (Zadnikova et al., 2010). These observations indicate that auxin levels above the optimum would inhibit growth at the inner side of the hook (Figure 2).

During apical hook development, the asymmetrical auxin gradient requires normal auxin synthesis (Figure 3). Mutations in the biosynthetic YUCCA flavin monooxigenases (Zhao et al., 2001), SUR1/SUR2 (SUPERROOT 1/2) (Boerjan et al., 1995; Delarue et al., 1998) or TAA1/TAR2 (TRYPTOPHAN AMINOTRANSFERASE 1-TRYPTOPHAN AMINOTRANSFERASE RELATED 2) (Stepanova et al., 2008; Vandenbussche et al., 2010) genes impair hook development. The 


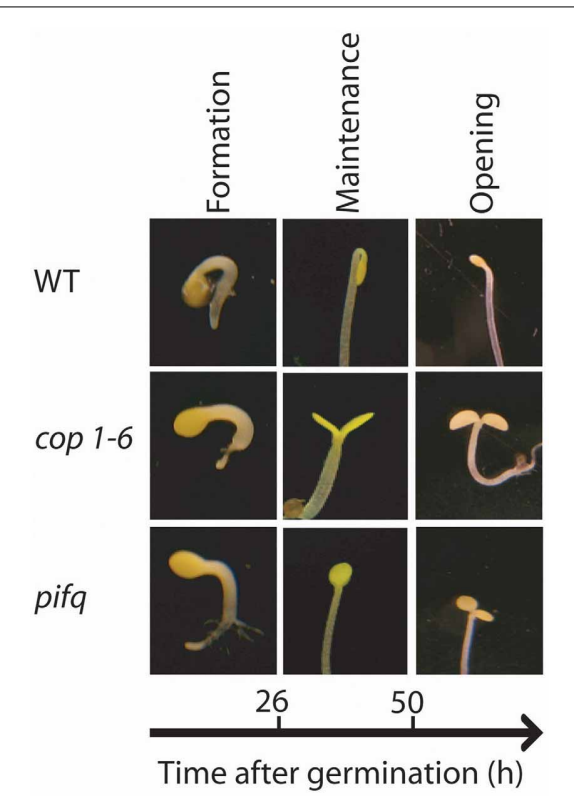

FIGURE 1 | Apical hook development in dark grown seedlings requires the repressors of photomorphogenesis COP1 and PIFs. Representative photographs of the apical section of the hypocotyls of WT Col, cop 1-6 and pifq mutants during the three phases of hook development. Photographs were taken $24 \mathrm{~h}$ after germination for the formation phase, $48 \mathrm{~h}$ after germination for the maintenance phase and $84 \mathrm{~h}$ after germination for the opening phase. dominant $y u c 1-D$ mutant contains elevated levels of free auxin and is hookless (Zhao et al., 2001; Vandenbussche et al., 2010). Although auxin synthesis is required for hook development, the auxin gradient is probably not the result of asymmetric auxin synthesis driven by the flavin monooxigenases, because the pattern of GUS activity driven by the YUC1 promoter is symmetric during hook formation and maintenance (Vandenbussche et al., 2010).

The auxin gradient depends largely on the correct localization of auxin carriers at the plasma membrane. Blocking auxin transport efflux or influx carriers, respectively, by 1$\mathrm{N}$-naphthylphthalamic acid (NPA) or 1-naphthoxyacetic acid (1-NOA) reduces hook formation (Vandenbussche et al., 2010; Zadnikova et al., 2010). Auxin is transported during hook development by the (PIN) (PIN-FORMED) auxin efflux carriers PIN1, PIN3, PIN4 and PIN7 (Zadnikova et al., 2010), the AUX1/LAX3 (AUXIN-RESISTANT 1/LIKE-AUX 13 ) auxin import carriers and the ABCB19 (ATP-binding cassette (ABC-type) subfamily B number 19 auxin efflux transporter) (Wu et al., 2010) (Figure 3). AUX1 would help to transfer auxin from the cotyledons to the hook; PIN3 carriers would move auxin from the endodermis to the cortex; AUX1, PIN3 and PIN4 from the cortex to the epidermis, and LAX3 would move auxin from the hook toward the roots (Vandenbussche et al., 2010; Zadnikova et al., 2010). PIN:GUS expression patterns show differentially spatial and temporal expression for PIN1, PIN3, PIN4, and PIN7 during apical hook development in darkness (Zadnikova et al., 2010). PIN3 exerts its main role during the formation and maintenance phases while PIN1, PIN4, and PIN7 are important during the
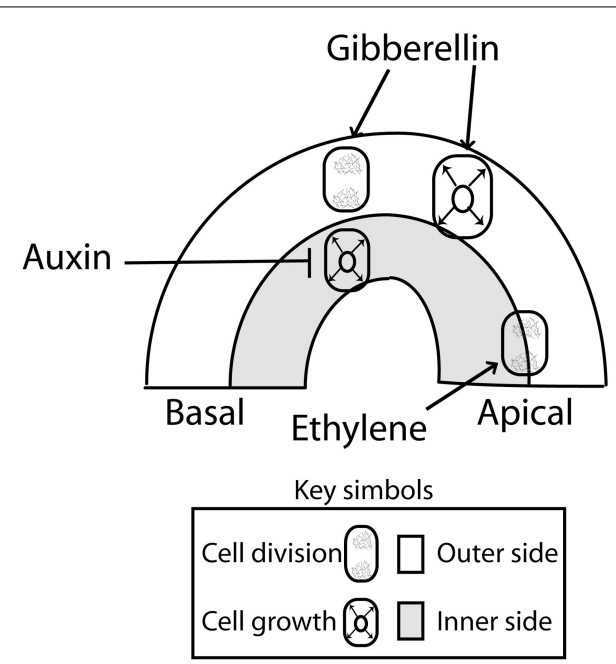

FIGURE 2 | Primary action of auxin (inhibition of cell expansion at the inner side), gibberellins (promotion of cell division and expansion at the outer side) and ethylene (enhanced cell division at the top) during apical hook development. There are interactions not represented here, such as the effects of gibberellins and ethylene on the auxin signaling gradient (see Figure 3). Auxin inhibition of cell growth at the inner side of the hook might be mediated by an enhanced ethylene signaling as auxin levels concentrations above a threshold might enhanced ethylene production and signaling contributing to cell growth inhibition (Abel et al., 1995; Raz and Ecker, 1999; Vandenbussche et al., 2010). Arrows: Positive regulation; T-bars: Negative regulation. maintenance phase and the maintenance to opening transition phases (Zadnikova et al., 2010). The pin3 (Zadnikova et al., 2010), aux1 and lax3 mutants (Vandenbussche et al., 2010) have severe defects in hook development suggesting these transporters have predominant roles in auxin transport in apical hooks. PIN3 is expressed in the hypocotyl zone where the curvature is maximal, mainly in the outer side of the hook. GFP-ABCB19 is present mostly at the inner side of the hook (Wu et al., 2010). During the opening phase, the enhanced expression at the inner side of the hook is reduced and the auxin asymmetry is lost. Thus, the joint action of these auxin transporters results in the establishment of an auxin gradient toward the inner side of the hook.

Auxin binds to co-receptor complexes formed by TIR1/ABFs F-box receptors (TRANSPORT INHIBITOR RESPONSE 1/AUXIN BINDING F BOX PROTEINS) and AUX/IAA (AUXIN/INDOL-3-ACETIC ACID) proteins (Calderón Villalobos et al., 2012). As result, the AUX/IAA proteins become ubiquitinated and targeted to degradation in the $26 \mathrm{~S}$ proteosome. AUX/IAA represses ARF (AUXIN RESPONSE FACTOR) transcription factors. Auxin releases ARFs from this inhibition and then ARF activators (such as ARF7 and ARF19) modulate the expression of auxin-responsive genes like the SAUR (SMALL AUXIN UP RNA) and GH3 (GRETCHEN HAGEN 3) gene families (reviewed by Calderon-Villalobos et al., 2010; Hayashi, 2012). Hook development requires normal auxin perception and signaling. Multiple mutants at receptor loci (Dharmasiri et al., 2005), gain-of-function iaal (Yang et al., 2004), iaa3, iaa12, iaa13 (Tian and Reed, 1999; Zadnikova et al., 


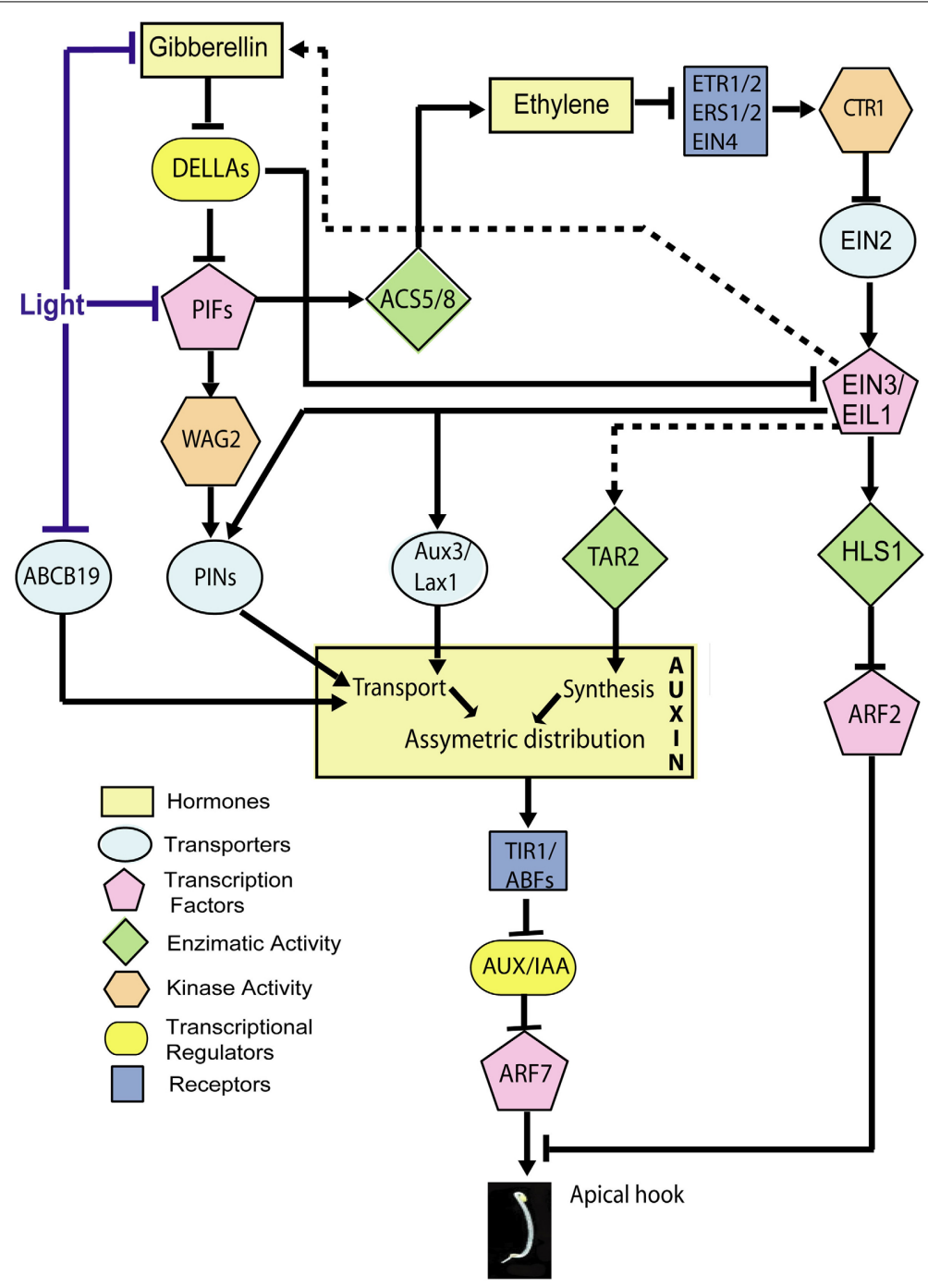

FIGURE 3 | Hormone signaling network involved in the inhibition of growth at the inner side of the apical hook by auxin. Established points of action of light during hook opening are indicated. The scheme includes only the components for which specific experimental evidence for a role in apical hook development is available (therefore, for instance, while GID1 should work between Gibberellins and
DELLA, this receptor is not included). Lines indicate direct or indirect connections between components. Arrows: Positive regulation; T-bars: Negative regulation. Dotted lines: The expression of TAR2 is promoted by ethylene and the abundance of gibberellins is promoted by ethylene in an EIN2-dependent manner, but whether these responses depend on EIN3 is not demonstrated.
2010) and iaa19 mutants (Tatematsu et al., 2004), loss of function nph4/arf7 and arf19 mutants (Harper et al., 2000; Zadnikova et al., 2010), lines expressing stabilized versions of the SAUR19-24 proteins (Spartz et al., 2012), and plants overexpressing SAUR32 (Park et al., 2007) have severe defects in apical hook formation or maintenance (Figure 3). In addition to SAUR and GH3 genes, auxin also induces the expression of some AUX/IAA genes. SAUR32, IAA3:GUS, IAA12:GUS and IAA13:GUS reporter lines express GUS activity differentially at the inner side of the hook (Park et al., 2007; Zadnikova et al., 2010).

The HLS1 (HOOKLESS 1) gene encodes a N-acetyltransferase that is required for apical hook formation. The $h l s 1$ mutant fails to form the apical hook due to defects in both differential cell elongation and cell division (the mutant does not express the
CycB1:GUS marker of mitotic division in the apical portion of the hypocotyl) (Lehman et al., 1996; Raz and Koornneef, 2001). HLS1 is actually required to establish the enhanced auxin-dependent gene expression at the inner side of the apical hook that in turn causes the asymmetric growth (Li et al., 2004).

In addition to the ARFs as activators in auxin-inducible gene expression, there are some ARFs with a negative action. ARF2 is a transcription factor that binds specifically to a DNA sequence present in auxin responsive promoter elements and represses auxin-induced expression (Tiwari et al., 2003) (Figure 3). ARF2 and ARF7 (negative and positive players in auxin signaling, respectively) might bind different promoter regions or might compete each other for the same promoter-binding sites in auxin responsive genes (Ulmasov et al., 1997, 1999; Vernoux et al., 
2011). ARF1 and ARF2 function redundantly as repressor of auxin action in apical hook formation (Li et al., 2004). HLS1 reduces the levels of ARF2 (Figure 3). Actually, the arf2 mutant was identified as a downstream extragenic suppressor of the $h l s 1$ mutation in dark-grown apical hook formation, which partially restores asymmetrical DR5:GUS expression (Li et al., 2004). ARF2 protein levels are not affected by auxin (Li et al., 2004); therefore, ARF2 appears to provide an auxin-independent repression of auxin-induced genes (Vernoux et al., 2011).

In summary, an asymmetrical auxin gradient in apical hooks leads to differential cell growth and hook formation and maintenance. The establishment of the auxin gradient requires normal auxin synthesis, transport, perception and signaling. The final result of auxin signaling on hook development would be mediated by the combined actions of activators and repressors ARFs on auxin-induced genes. Together with auxin, gibberellins, ethylene and brassinosteroids contribute to hook formation and are discuss in the following sections.

\section{NORMAL HOOK DEVELOPMENT REQUIRES GIBBERELLINS}

Gibberellins are important during the initial phase and determine the speed and the degree of hook formation (Gallego-Bartolomé et al., 2011). Seedlings treated with an inhibitor of gibberellins biosynthesis show decreased DR5:GUS expression at the inner side of the hook but the deficient hook of the pin 3 pin 7 double mutant cannot be restored by gibberellins (Gallego-Bartolomé et al., 2011). These observations indicate that gibberellins act in part by favoring the generation of a normal auxin gradient (Figure 3). In addition, gibberellins appear to enhance apical hook formation more directly, by promoting cell elongation and cell division at the outer side of the hook (Vriezen et al., 2004) (Figure 2). This suggests that the hook is formed by the auxin-mediated inhibition of growth at the inner side and the gibberellin-mediated promotion of growth at the outer side.

Hook formation requires gibberellins to reduce the abundance of DELLAs (Alabadí et al., 2004; Gallego-Bartolomé et al., 2011) (Figure 3). DELLAs are nuclear proteins of the GRAS family (Bolle, 2004), which repress growth. When gibberellins bind the receptor GID1 (GIBBERELIN INSENSITIVE DWARF 1) the latter forms a complex that catalyses ubiquitination of DELLA, leading to their rapid degradation and the release of growth (Ueguchi-Tanaka et al., 2007; Willige et al., 2007; Ariizumi et al., 2008). Five DELLA genes are present in Arabidopsis (GAI, RGA, RGL1, RGL2, and RGL3) (Peng et al., 1997; Silverstone et al., 1998). The rga and gai loss of function mutations suppress the hookless phenotype of 3-d-old etiolated gibberellin deficient ga13 mutants (Achard et al., 2003). The quintuple della mutant shows a steeper slope of hook angle formation than the WT during the first day after germination, but thereafter it behaves similarly to the WT. These results suggest that gibberellins requirement during apical hook development is important during the formation and maintenance phases (Gallego-Bartolomé et al., 2011).

The quintuple della mutant displays closed hooks while the hls1 della mutant is completely hookless suggesting that the effects of gibberellins and DELLA depend on HLS1 (An et al., 2012). Gibberellins promote HLS1 expression (Gallego-Bartolomé et al., 2011; An et al., 2012) and the gai-1 mutant allele, that encodes a dominant version of DELLA protein GAI, reduces HLS1 expression (Gallego-Bartolomé et al., 2011). DELLA proteins directly interact with the DNA binding domain of EIN3/EIL1 (ETHYLENE INSENSITIVE 3/EIN3 LIKE 1), repressing its function (Figure 3). EIN3/EIL1 are transcription factors that interact directly with the HLS1 promoter to activate its transcription (An et al., 2012) (Figure 3). Thus, gibberellins release EIN3/EIL1 from DELLA inhibition to promote HLS1 expression (An et al., 2012).

In addition, DELLAs interact directly with PIF3 (PHYTOCHROME INTERACTING FACTOR 3) and PIF4 (see Box 1), interfering with their ability to bind their target gene promoters (De Lucas et al., 2008; Feng et al., 2008) (Figure 3). PIFs are helix-loop-helix transcription factors and at least PIF1, PIF3, and PIF5 promote hook development in darkness (Khanna et al., 2007; Leivar et al., 2008; Gallego-Bartolomé et al., 2011). Gibberellins and at least PIF1 expression in the endodermis are required for hook formation (Gallego-Bartolomé et al., 2011; Kim et al., 2011). The connection between the gibberellin-DELLA-PIF module and the auxin gradient would be established by WAG2 (a member of the AGC3 kinase subclass family) (Willige et al., 2012). WAG2 is expressed in a gibberellin-dependent manner at the inner side of the apical hook, and PIF5 binds to a G-box of the WAG2 promoter increasing its expression (Willige et al., 2012) (Figure 3). WAG2 is an auxin transport-regulatory protein kinase that phosphorylates in vitro the central intracellular loop of PIN1, PIN3, PIN4, and PIN7 proteins (Willige et al., 2012) (Figure 3). Four day-old dark-grown wag2 mutant seedlings display more open hooks than WT seedlings, but 2-d-old darkgrown seedlings are indistinguishable from the WT, suggesting WAG2 is important during apical hook maintenance and the repression of apical hook opening but not during apical hook formation.

So, gibberellins are crucial for apical hook formation. Gibberellins are needed to maintain DELLA levels reduced and thus PIF and EIN3/EIL1 transcription factors are available to increase WAG2 and HLS1 which regulate asymmetric auxin accumulation.

\section{ETHYLENE PROMOTES AUXIN SYNTHESIS, TRANSPORT AND SIGNALING AT THE INNER SIDES OF APICAL HOOKS}

Dark-grown seedlings treated with exogenous ethylene exhibit an exaggerated apical hook, which is one of the components of the classical triple response. In addition, an exaggerated hook is observed in dark-grown dominant mutants that display elevated levels of ethylene, like ethylene overproducer mutants (single mutants eto1, eto2, and eto3) (Guzman and Ecker, 1990; Vogel et al., 1998b; Woeste et al., 1999); and reduced hook curvature is observed in mutants that fail to increase ethylene biosynthesis like cytokinin insensitive (single mutants $\operatorname{cin} 1, \operatorname{cin} 2, \operatorname{cin} 3$, and $\operatorname{cin} 4$ ) (Vogel et al., 1998a). Furthermore, eto2 is a dominant mutation of the ethylene biosynthesis 1-aminocyclopropane-1-carboxylate synthase ACS5 gene (Vogel et al., 1998b). Thus, ethylene biosynthesis is important in the regulation of hook curvature. Ethylene has a role during the formation phase and a predominant role in hook maintenance by controlling cell division mainly along the apical-basal parts of the hooks (Raz and Koornneef, 2001) (Figure 2). 
Ethylene is perceived by the ETR1, ETR2 (ETHYLENE RESISTANT 1 AND 2), ERS1, ERS2 (ETHYLENE RESPONSE SENSOR 1 AND 2) and EIN4 (ETHYLENE INSENSITIVE 4) receptors (Bleecker, 1999; Schaller and Kieber, 2002). In the absence of ethylene, the receptors are active (Hua and Meyerowitz, 1998) (Figure 3). Dominant mutations in these receptors (like single mutants etr1-1, ers1-1) confer ethylene insensitivity and lack of an apical hook, while loss-of-function mutations (like single mutants etr1-7, etr2-3, ein4-7) cause hypersensitivity to ethylene and exaggerated hooks after ethylene treatment (Hua et al., 1998; Sakai et al., 1998; Raz and Ecker, 1999; Qu et al., 2007; Liu and Wen, 2012). These receptors activate CTR1 (CONSTITUTIVE TRIPLE RESPONSE 1) (Clark et al., 1998), which negatively regulates EIN2 (ETHYLENE INSENSITIVE 2) and EIN3/EIL1 (Figure 3). The ctr1 mutant displays an exaggerated hook phenotype (Knee et al., 2000).

In addition to its effects on cell division, ethylene also acts indirectly by inducing auxin transport and increasing auxin levels at the inner side of the hook (De Grauwe et al., 2005; Zadnikova et al., 2010) via different pathways that target auxin synthesis, auxin transport and auxin-induced gene expression (Figure 3).

Ethylene enhances the expression of the auxin synthesis gene TAR2 at the inner side of the hook 3 days after germination (Vandenbussche et al., 2010) (Figure 3). In addition, EIN3 binds the promoter of ASA1 (ALPHA SUBUNIT OF ANTHRANILATE SYNTHASE 1), the enzyme that catalyzes the first limiting step in tryptophan synthesis (Chang et al., 2013). Auxin accumulation in the root tips is promoted by ethylene through the promotion of ASA1 expression, and the GUS activity of the transcriptional reporter ASA1:GUS is not enhanced by ethylene in an ein2 mutant (Stepanova et al., 2005). Thus, it is reasonable to speculate that ethylene effects on TAR2 are mediated by EIN3.

Ethylene enhances the expression of PIN1, PIN3, PIN4, and PIN7 auxin efflux carriers (Zadnikova et al., 2010) and AUX1 and LAX1 auxin influx carriers (Vandenbussche et al., 2010) (Figure 3). The hookless phenotype of the pin3 mutant seedlings displays reduced sensitivity to ethylene (Zadnikova et al., 2010). NPA treatments almost completely suppress the exaggerated hook phenotype in ctr1 mutants and EIN3 overexpressor seedlings (An et al., 2012), suggesting auxin functions downstream of EIN3/EIL1. Also, EIN3 binds PIN7 and AUX1 promoters (Chang et al., 2013) (Figure 3). Thus, ethylene would modulate auxin distribution by controlling the expression of auxin transporters through EIN3/EIL1 transcription factors. Modulation of auxin transport in apical hooks is a common event regulated by ethylene and gibberellin actions (Figure 3).

Ethylene promotes HLS1 expression (Lehman et al., 1996; Du and Kende, 2001; Li et al., 2004; An et al., 2012) (Figure 3) and HLS1 abundance (Li et al., 2004) and also reduces ARF2 abundance (Li et al., 2004). The hls1 mutant remains hookless even after being treated with ethylene (Li et al., 2004). The loss of phototropic response in the arf7 mutant can be compensated by ethylene treatment but not that of the arf7 hls1 double mutant (Harper et al., 2000). This suggests that ethylene would favor the action of additional activating ARFs on auxin signaling by repressing the action of ARF2 via HLS1.
Ethylene also promotes gibberellins synthesis (Vriezen et al., 2004). Treatment with an ethylene precursor enhances the expression of the gibberellin biosynthetic gene GA1 in apical hooks of 3-d-old dark-grown seedlings (Vriezen et al., 2004) and the expression of a gibberellin responsive promoter fragment GASA1 at the outer side of the hook (Vriezen et al., 2004) (Figure 3). The promotion of GA1 expression by ethylene is not observed in the ein2 mutant (Vriezen et al., 2004). Even though the expression of GA1 gene by ethylene has not been tested in an ein 3 mutant, from the above results we speculate that gibberellin synthesis might be affected downstream EIN3/EIL1 ethylene regulator.

In addition to the effects of ethylene on gibberellins there is evidence for a reciprocal control. Ethylene production is enhanced in della mutants (Gallego-Bartolomé et al., 2011). PIF5 binds a G-box in the promoter of ACS 8 and induces its transcription in a gibberellin and DELLA-dependent manner (Gallego-Bartolomé et al., 2011) (Figure 3).

The previous paragraphs describe the pathways by which ethylene affects the asymmetric auxin-induced responses. Ethylene could also act downstream auxin, which might stimulate ethylene production or ethylene sensitivity at the inner side of the hooks (Raz and Ecker, 1999). In pea epicotyls, the transcript levels of the PsACO1, (the isoform of an ACC oxidase) are higher in the inner than in the outer side (Peck et al., 1998). Although this hypothesis has been largely accepted, it is not clear how ethylene would inhibit cell growth at the inner side of the hook.

In summary, normal ethylene production and signaling is necessary for the establishment of the apical hook. Ethylene signaling controls the responses of transcription of several genes through EIN3/EIL1 transcription factors integrating ethylene with gibberellin and auxin in apical hooks.

\section{BRASSINOSTEROIDS ARE REOUIRED FOR HOOK FORMATION}

Mutants defective in brassinosteroid synthesis such as det2 (deetiolated 2) lack an apical hook in darkness (Chory et al., 1991). Adding brassinosteroids or inhibitors of brassinosteroid synthesis alters the patterns of auxin response in the hook (De Grauwe et al., 2005) highlighting the function of this hormone during hook formation.

Brassinosteroids enhance the activity of the kinase BIN2 (BRINSENSITIVE 2) that phosphorylates ARF2 and reduces its activity, leading to the enhanced expression of auxin responsive genes (Vert et al., 2008). Some studies suggest that ethylene could control the apical hook partially by activating brassinosteroid biosynthesis (De Grauwe et al., 2005) or downstream signaling components (Gendron et al., 2008). Analysis of dark-grown seedlings carrying a CPD:GUS (CONSTITUTIVE PHOTOMORPHOGENESIS AND DARFWISM) reporter gene which is involved in brassinolide synthesis, shows induced expression of the reporter gene by ethylene in apical hooks. Also, darkgrown det 2 mutant seedlings treated with exogenous ethylene are not able to induce an exaggerated hook suggesting ethylene action requires brassinosteroids (De Grauwe et al., 2005). The double mutant bri1 bzr1-1D (brassinosteroid insensitive 1; brassinazole resistant 1) that is deficient in the perception of brassinosteroids but have activated downstream brassinosteroids responses due to 
a dominant mutation that stabilizes BZR1, forms and apical hook similar to that of the WT (Gendron et al., 2008). Thus, ethylene might also act regulating brassinosteroid downstream signaling. It has been proposed that brassinosteroids affect auxin distribution through PIN modulation (De Grauwe et al., 2005), however further work is necessary to define the points of regulation of brassinosteroids in the network signaling that leads to apical hook formation.

\section{LIGHT PERCEIVED BY PHYTOCHROMES AND CRYPTOCHROMES INDUCES HOOK OPENING}

Apical hook opening is one of the responses affected by the switch from skotomophogenic to photomorphogenic development. In most dicotyledonous plants, including Gossypium hirsutum L., Phaseolus vulgaris L., (Powell and Morgan, 1970) Pisum sativum (Britz and Galston, 1982) and A. thaliana (Liscum and Hangarter, 1993a), light induces apical hook opening. In some species, like Solanum Lycopersicum, characterized by a "hard to split" seed coat, light exaggerates hook formation possible as part of a mechanism that facilitates seed coat removal when seedlings germinate below the soil (Shichijo et al., 2010).

When A. thaliana seedlings are exposed to light, the hook structure opens completely within approximately $6 \mathrm{~h}$ (Liscum and Hangarter, 1993a; Wang et al., 2009). Blue, far-red and red light induce hook opening via the partially overlapping action of phytochromes and cryptochromes (Liscum and Hangarter, 1993a,b) (see Box 1; Figure 4). In hook opening of $A$. thaliana, red light is the least effective of these colors and its effect is mediated by the redundant actions of phytochrome A (phyA) and phyB (Reed et al., 1994). Far-red light perceived by phyA has a much stronger effect on the apical hook (Liscum and Hangarter, 1993b). Finally, the strong stimulation of hook opening by blue light appears to be mediated by cryptochromes 1 (cryl) in same cases (Liscum and Hangarter, 1993b), but not in others (Wang et al., 2009; Fox et al., 2012). In these cases blue light effects could be mediated by phyA (Casal and Mazzella, 1998; Neff and Chory, 1998; Chun et al., 2001). No function for other phytochromes (phyC, phyD, or phyE) or cry2 has been described for apical hook opening.

In addition to their role under specific wavelengths, photoreceptors are able to affect some processes in darkness downstream of light signals that were perceived at the seed stage, adjusting young seedlings to the environment they will likely have to face (Mazzella et al., 2005). cry1 mutants grown in darkness on agar containing sucrose display more opened hooks than WT seedlings at late stages of hook development, a phenotype also observed in mutants of the heterotrimeric G alpha subunit protein (GPA1) (Fox et al., 2012).

In apical hook cells of dark-grown pea, immunochemically detectable PHYA is distributed diffusely in the cytosol and appears in the nucleus upon exposure to continuous far-red or red light (Hisada et al., 2000) (see Box 1; Figure 4). In 8-dold, dark-grown soybean seedlings, $1 \mathrm{~h}$ far-red light enhances the expression of genes mostly involved in cell division and protein turnover in the hook itself (Li et al., 2011), indicating that the phytochrome signal reaches the nucleus. The translocation of phyA to the nucleus requires FHY1 (FAR RED ELONGATED HYPOCOTYL 1) and FHL1 (FAR RED
ELONGATED HYPOCOTYL LIKE 1) (Hiltbrunner et al., 2006; Rosler et al., 2007). The fhy1 mutant is impaired in apical hook opening under far-red light (Hiltbrunner et al., 2006) suggesting that far-red-induced apical hook opening needs phyA in the nucleus.

Thus, light triggers apical hook opening through the action of photoreceptors phyA phyB and cryl. This process must imply the disruption of the auxin gradient that leads to differentially cell growth. Downstream targets of photoreceptors involved in hook development will be discussed in the next sections.

\section{LIGHT REDUCES THE ACTIVITY OF PIFs AND COP1, WHICH ARE REOUIRED FOR HOOK DEVELOPMENT IN DARKNESS}

Photomorphogenesis is a process repressed in darkness principally by two pathways (Box 1; Figure 4). One involves the E3 ubiquitin ligase COP1 (CONSTITUTIVE PHOTOMORPHOGENIC 1) and the other involves the group of PIF transcription factors (Leivar and Quail, 2011; Lau and Deng, 2012) (see Box 1; Figure 4). The cop1 mutant (Deng et al., 1992; Alabadí et al., 2008) and the pif1 pif3 pif4 pif5 quadruple mutant (pifq) (Leivar et al., 2008; Shin et al., 2009) have a de-etiolated phenotype when grown in darkness. Both copl and pifq are not able to form an apical hook (Figure 1). Thus, COP1 and PIF proteins are indispensable for hook formation. In the presence of light, at least phyA, phyB, and cryl activities repress COP1 protein while phyA and phyB repress PIF proteins (see Box 1; Figure 4).

\section{DOWNSTREAM OF PIFs AND COP1}

Apical hook formation implies the establishment of an auxin signaling maxima and the asymmetric distribution of auxin at the apical hook but this asymmetry has to be compensated during the opening phase. DR5 expression signals are very strong in the concave side of the dark-grown seedlings, but this asymmetry is greatly reduced or even disappears during the opening phase either in the dark (Zadnikova et al., 2010; Willige et al., 2012) or upon exposure to light (Wu et al., 2010). PIFs have several important points of action leading to the generation of the auxin gradient and hook development in darkness (Figure 3). Therefore, the reduction of PIF activity by phytochromes (Box 1; Figure 4) is predicted to affect the auxin gradient via these pathways. In support of this view, light-induced changes have been documented for some elements that would operate downstream of PIFs in darkness. For instance, WAG2 transcript levels decrease (Willige et al., 2012) (Figure 3), HLS1 transcript levels also decrease and ARF2 protein levels increase after light exposure (Li et al., 2004). As expected, ARF2 accumulation was not observed in light exposed hls1 mutants (Li et al., 2004) (Figure 3). Ethylene sensitivity and/or responses in the apical hook are attenuated by phyA, phyB and cryl activation (Knee et al., 2000), so phytochrome-mediated modulation might involve the control of ACS 8 expression by PIF5 (Figure 3).

The reduction of PIF3 levels by red light has other consequences. For instance, $1 \mathrm{~h}$ after the beginning of red light, low PIF3 activity reduces the expression of the $B B X 23$ gene (a member of the B-box family of transcription factors). Since 


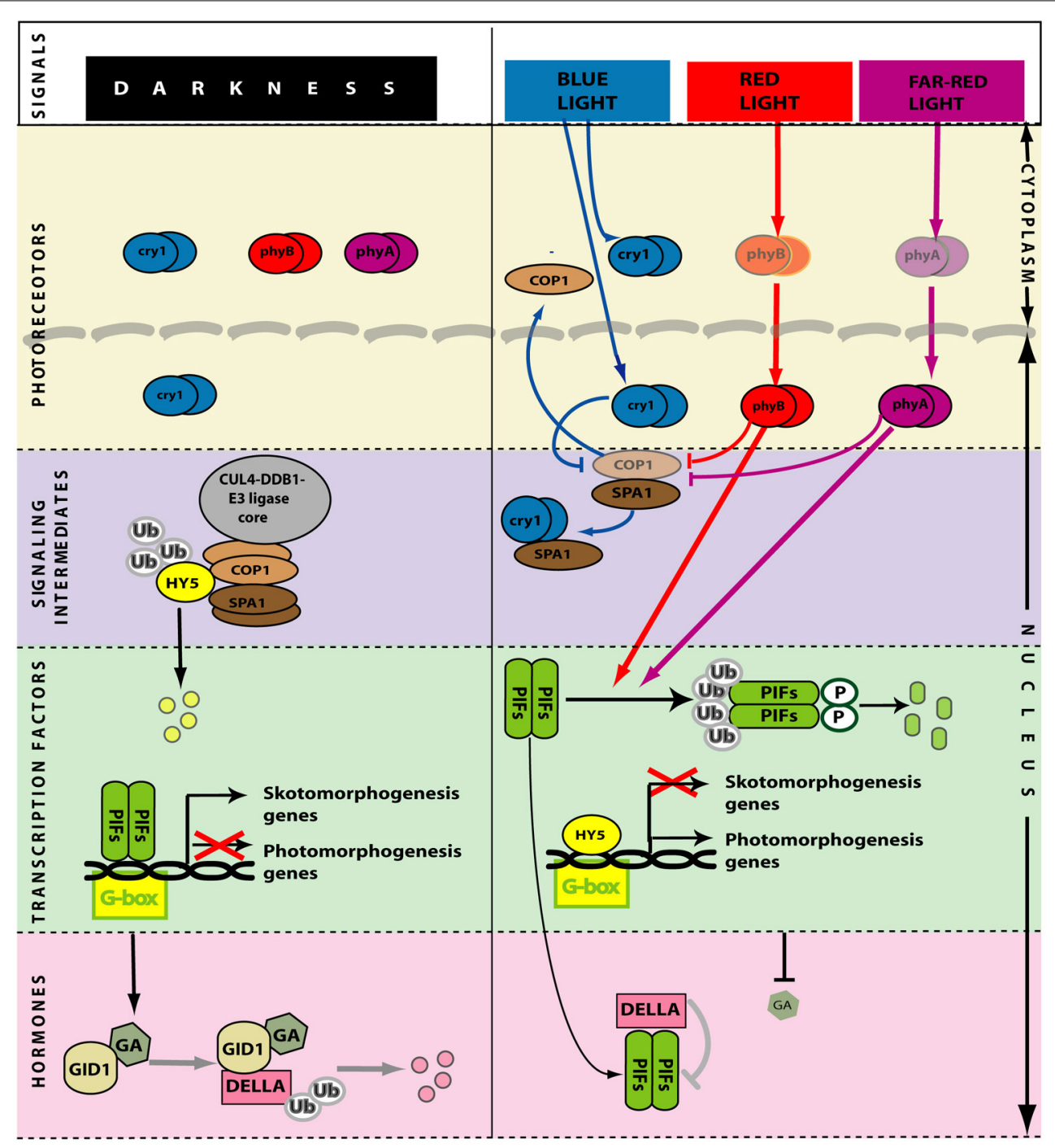

FIGURE 4 | Simplified model of light perception and signaling during de-etiolation. In darkness (left) the photoreceptors are inactive. PIF transcription factors promote skotomorphogenesis. HY5 and other transcription factors that promote photomorphogenesis are

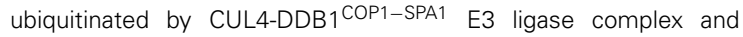
degraded in the $26 \mathrm{~S}$ proteasome. High levels of gibberellins induce degradation of DELLA. Light (right) activates the photoreceptors. In the nucleus, phytochromes (that migrate from the cytoplasm in their active form) and cryptochromes interact with COP1, reducing its activity and allowing the abundance of HY5 to increase. In the nucleus, phytochromes also reduce the activity of PIFs. Gibberellin levels are reduced, DELLA accumulate and bind PIFs further reducing their activity. GA, gibberellins. Arrows: Positive regulation; T-bars: Negative regulation.
BBX23 is a repressor of photomorphogenesis required for hook maintenance in darkness, down-regulation of its expression enhances hook opening (Sentandreu et al., 2011). At later stages, (3-h after the beginning of red light) the reduced PIF3 levels favor the induction of expression of $P P 2 C$ (a type C phosphatase), which attenuates late hook opening possibly as part of a mechanism that avoids an exaggerated response of hook opening to light stimulus once seedlings are de-etiolated (Sentandreu et al., 2011).

In darkness, COP1 directly ubiquitinates and targets to degradation in the $26 \mathrm{~S}$ proteasome several transcription factors required for photomorphogenesis, including HY5 (LONG HYPOCOTYL 5) (see Box 1; Figure 4). BBX23 is also ubiquitinated by COP1 (Datta et al., 2008) and could therefore represent a point of convergence between COP1 and PIFs. Mutations in transcription factors genes downstream of COP1 signaling like hyh (hy5 homolog) show impaired hook opening under far-red light, but hfrl (long hypocotyl in far-red) and laf1 (long after far-red light) mutants are affected in hypocotyl elongation but not in hook opening under far-red light (Fairchild et al., 2000; Ballesteros et al., 2001). These data support the idea that, even though the apical hook structure is a part of the hypocotyl, hook formation and hypocotyl straight growth are two processes regulated independently downstream of COP1. Also, COP1 is able to regulate the amount of EIN3/EIL1 proteins during photo-oxidative damage (Zhong et al., 2009) and hypocotyl growth responses (Liang et al., 2012) playing crucial roles in 
ethylene signaling. It would be interesting to determine if COP1 regulates EIN3/EIL1 during hook development.

Light decreases the level of gibberellins in Arabidopsis (AitAli et al., 1999; Achard et al., 2007; Alabadí et al., 2008) pea (Weller et al., 2009) and rice (Hirose et al., 2012) (Figures 3, 4). Blue light acting through cryl reduces the expression of genes involved in gibberellin biosynthesis and the levels of this hormone (Folta et al., 2003; Foo et al., 2006). In addition, the pea HY5 gene induces the expression of gibberellins-2-oxidase, the major gibberellin catabolic enzyme in plants, resulting in negatively regulation of gibberellin pathways (Weller et al., 2009). Additionally, phyA and phyB activities increase the accumulation of DELLA proteins (Achard et al., 2007). As mentioned before, EIN3/EIL1 are direct targets of DELLA proteins, which inhibits their function affecting their downstream targets elements. So, light might reduced EIN3/EIL1 availability through its dual effects on PIFs and on DELLA proteins (Figure 3). It has been shown that gibberellin signaling deficiencies in the cop 1 mutant of several species (Alabadí et al., 2008; Weller et al., 2009; Tanaka et al., 2011) suggesting that COP1 might be necessary for establishing gibberellins levels in darkness for hook formation.

In the hooks, light also affects the expression and localization of the auxin transporter ABCB19. ABCB19 localizes to the inner side of etiolated hooks but its presence fades away during hook opening under blue light (Wu et al., 2010) (Figure 3). Red or blue light perceived by phyA, phyB, and/or cryl reduce ABCB19 protein levels in the upper section of the hypocotyls (Nagashima et al., 2008). There might be additional effects of light on auxin transporters during hook opening as at least during phototropic responses, light causes changes in the subcellular distribution of selected PIN carriers in the hypocotyls (Blakeslee et al., 2004; Ding et al., 2011). PIN1 in the hypocotyls and PIN1 and PIN2 intracellular distribution and abundance in the roots are light-regulated and controlled by COP1 (Sassi et al., 2012).

In summary, COP1 and PIFs are direct key targets of light signaling to control hook opening. PIFs operate downstream of gibberellins and affect auxin transport and ethylene synthesis. EIN3/EIL1 are indirect targets of light signaling, through the PIFs and DELLA pathways, operate downstream gibberellins and ethylene and affect auxin transport, synthesis and signaling. The connections between COP1 and the hormonal network that underlies the formation of the hook in darkness are not firmly established.

\section{CONCLUDING REMARKS}

The coordinated actions of gibberellins, ethylene and brassinosteroids control asymmetric auxin distribution to allow the correct development of the hook in darkness. This control occurs at multiple levels, including synthesis, distribution by the action of specific transporters and abundance of transcription factors involved in the control of auxin-responsive genes. PIFs and EIN3/EIL1 are key connectors of gibberellins and ethylene pathways with auxin signaling in the hooks. The repressors of photomorphogenesis PIFs and COP1 are indispensable for hook development in darkness and the activation of phytochromes and cryptochromes by light negatively regulates the activity of PIFs and COP1, leading to the opening of the hook.
Considerable progress has been made in recent years in the elucidation of the signaling network that controls apical hook formation in darkness; however there still are several gaps concerning how light-regulated proteins modulate hook formation and triggers hook opening. Learning the dynamics of the hormonal networks in response to light is a challenge for future research.

\section{ACKNOWLEDGMENTS}

This work was supported by PIP CONICET 037 and PICT 2010 1821 to Maria A. Mazzella and by PICT 20101819 and UBACYT to Jorge J. Casal and PICT 20111689 to Jorge P. Muschietti.

\section{REFERENCES}

Abel, S., Nguyen, M. D., Chow, W., and Theologis, A. (1995). Asc4, a primary indoleacetic acid-responsive gene encoding 1-aminocyclopropane-1carboxylate synthase in Arabidopsis thaliana: structural characterization, expression in Escherichia coli, and expression characteristics in response to auxin. J. Biol. Chem. 270, 19093-19099. doi: 10.1074/jbc.270.32.19093

Achard, P., Liao, L., Jiang, C., Desnos, T., Bartlett, J., Fu, X., et al. (2007). DELLAs contribute to plant photomorphogenesis. Plant Physiol. 143, 1163-1172. doi: 10.1104/pp.106.092254

Achard, P., Vriezen, W. H., Van Der Straeten, D., and Harberd, N. P. (2003). Ethylene Regulates Arabidopsis Development via the Modulation of DELLA Protein Growth Repressor Function. Plant Cell 15, 2816-2825. doi: 10.1105/tpc.015685

Ait-Ali, T., Frances, S., Weller, J. L., Reid, J. B., Kendrick, R. E., and Kamiya, Y. (1999). Regulation of gibberellin 20-oxidase and gibberellin 3beta-hydroxylase transcript accumulation during De-etiolation of pea seedlings. Plant Physiol. 121, 783-791. doi: 10.1104/pp.121.3.783

Alabadí, D., Gallego-Bartolomé, J., Orlando, L., Garcia-Carcel, L., Rubio, V., Martinez, C., et al. (2008). Gibberellins modulate light signaling pathways to prevent Arabidopsis seedling de-etiolation in darkness. Plant J. 53, 324-335. doi: 10.1111/j.1365-313X.2007.03346.x

Alabadí, D., Gil, J., Blázquez, M. A., and Garcia-Martinez, J. L. (2004). Gibberellins repress photomorphogenesis in darkness. Plant Physiol. 134, 1050-1057. doi: 10.1104/pp.103.035451

Al-Sady, B., Ni, W., Kircher, S., Schafer, E., and Quail, P. H. (2006). Photoactivated phytochrome induces rapid PIF3 phosphorylation prior to proteasome-mediated degradation. Mol. Cell. 23, 439-446. doi: 10.1016/j.molcel.2006.06.011

An, F., Zhang, X., Zhu, Z., Ji, Y., He, W., Jiang, Z., et al. (2012). Coordinated regulation of apical hook development by gibberellins and ethylene in etiolated Arabidopsis seedlings. Cell Res. 22, 915-927. doi: 10.1038/cr.2012.29

Ariizumi, T., Murase, K., Sun, T. P., and Steber, C. M. (2008). Proteolysisindependent downregulation of DELLA repression in Arabidopsis by the gibberellin receptor GIBBERELLIN INSENSITIVE DWARF1. Plant Cell 20, 2447-2459. doi: 10.1105/tpc.108.058487

Bai, M. Y., Shang, J. X., Oh, E., Fan, M., Bai, Y., Zentella, R., et al. (2012). Brassinosteroid, gibberellin and phytochrome impinge on a common transcription module in Arabidopsis. Nat. Cell Biol. 14, 810-817. doi: 10.1038/ ncb2546

Ballesteros, M. L., Bolle, C., Lois, L. M., Moore, J. M., Vielle-Calzada, J. P., Grossniklaus, U., et al. (2001). LAF1, a MYB transcription activator for phytochrome A signaling. Genes Dev. 15, 2613-2625. doi: 10.1101/gad.915001

Blakeslee, J. J., Bandyopadhyay, A., Peer, W. A., Makam, S. N., and Murphy, A. S. (2004). Relocalization of the PIN1 auxin efflux facilitator plays a role in phototropic responses. Plant Physiol. 134, 28-31. doi: 10.1104/pp.103.031690

Bleecker, A. B. (1999). Ethylene perception and signaling: an evolutionary perspective. Trends Plant Sci. 4, 269-274. doi: 10.1016/S1360-1385(99)01427-2

Boerjan, W., Cervera, M. T., Delarue, M., Beeckman, T., Dewitte, W., Bellini, C., et al. (1995). Superroot, a recessive mutation in Arabidopsis, confers auxin overproduction. Plant Cell 7, 1405-1419. doi: 10.1105/tpc.7.9.1405

Bolle, C. (2004). The role of GRAS proteins in plant signal transduction and development. Planta 218, 683-692. doi: 10.1007/s00425-004-1203-Z 
Britz, S. J., and Galston, A. W. (1982). Physiology of Movements in Stems of Seedling Pisum sativum L. cv Alaska: II. the role of the apical hook and of auxin in nutation. Plant Physiol. 70, 1401-1404. doi: 10.1104/pp.70.5.1401

Calderón Villalobos, L. I., Lee, S., De Oliveira, C., Ivetac, A., Brandt, W., Armitage, L., et al. (2012). A combinatorial TIR1/AFB-Aux/IAA co-receptor system for differential sensing of auxin. Nat. Chem. Biol. 8, 477-485. doi: 10.1038/nchembio. 926

Calderon-Villalobos, L. I., Tan, X., Zheng, N., and Estelle, M. (2010). Auxin perception-structural insights. Cold Spring Harb. Perspect. Biol. 2, a005546. doi: 10.1101/cshperspect.a005546

Casal, J. J., Candia, A. N., and Sellaro, R. (2013). Light perception and signalling by phytochrome A. J Exp Bot. doi: 10.1093/jxb/ert379. [Epub ahead of print].

Casal, J. J., Fankhauser, C., Coupland, G., and Blázquez, M. A. (2004). Signalling for developmental plasticity. Trends Plant Sci. 9, 309-314. doi: 10.1016/j.tplants.2004.04.007

Casal, J. J., and Mazzella, M. A. (1998). Conditional synergism between cryptochrome 1 and phytochrome B is shown by the analysis of phyA, phyB, andhy 4 simple, double, and triple mutants in Arabidopsis. Plant Physiol. 118, 19-25. doi: $10.1104 /$ pp.118.1.19

Cashmore, A. R., Jarillo, J. A., Wu, Y. J., and Liu, D. (1999). Cryptochromes: blue light receptors for plants and animals. Science. 284, 760-765. doi: 10.1126/science.284.5415.760

Chang, K. N., Zhong, S., Weirauch, M. T., Hon, G., Pelizzola, M., Li, H., et al. (2013). Temporal transcriptional response to ethylene gas drives growth hormone cross-regulation in Arabidopsis. Elife. 2, e00675. doi: 10.7554/eLife.00675

Chen, D., Xu, G., Tang, W., Jing, Y., Ji, Q., Fei, Z., et al. (2013). Antagonistic basic helix-loop-helix/bZIP transcription factors form transcriptional modules that integrate light and reactive oxygen species signaling in Arabidopsis. Plant Cell 25, 1657-1673. doi: 10.1105/tpc.112.104869

Chory, J., Nagpal, P., and Peto, C. A. (1991). Phenotypic and genetic analysis of det2, a new mutant that affects light-regulated seedling development in Arabidopsis. Plant Cell 3, 445-459. doi: 10.1105/tpc.3.5.445

Chun, L., Kawakami, A., and Christopher, D. A. (2001). Phytochrome A mediates blue light and UV-A-dependent chloroplast gene transcription in green leaves. Plant Physiol. 125, 1957-1966. doi: 10.1104/pp.125.4.1957

Clark, K. L., Larsen, P. B., Wang, X., and Chang, C. (1998). Association of the Arabidopsis CTR1 Raf-like kinase with the ETR1 and ERS ethylene receptors. Proc. Natl. Acad. Sci. U.S.A. 95, 5401-5406. doi: 10.1073/pnas.95.9.5401

Datta, S., Johansson, H., Hettiarachchi, C., Irigoyen, M. L., Desai, M., Rubio, V., et al. (2008). LZF1/SALT TOLERANCE HOMOLOG3, an Arabidopsis B-box protein involved in light-dependent development and gene expression, undergoes COP1-mediated ubiquitination. Plant Cell 20, 2324-2338. doi: 10.1105/tpc. 108.061747

De Grauwe, L., Vandenbussche, F., Tietz, O., Palme, K., and Van Der Straeten, D. (2005). Auxin, ethylene and brassinosteroids: tripartite control of growth in the Arabidopsis hypocotyl. Plant Cell Physiol. 46, 827-836. doi: 10.1093/pcp/pci111

Delarue, M., Prinsen, E., Onckelen, H. V., Caboche, M., and Bellini, C. (1998). Sur2 mutations of Arabidopsis thaliana define a new locus involved in the control of auxin homeostasis. Plant J. 14, 603-611. doi: 10.1046/j.1365-313X.1998.00163.x

De Lucas, M., Daviere, J. M., Rodriguez-Falcon, M., Pontin, M., Iglesias-Pedraz, J. M., Lorrain, S., et al. (2008). A molecular framework for light and gibberellin control of cell elongation. Nature. 451, 480-484. doi: 10.1038/ nature 06520

Deng, X. W., Matsui, M., Wei, N., Wagner, D., Chu, A. M., Feldmann, K. A., et al. (1992). COP1, an Arabidopsis regulatory gene, encodes a protein with both a zinc-binding motif and a G beta homologous domain. Cell. 71, 791-801. doi: 10.1016/0092-8674(92)90555-Q

Dharmasiri, N., Dharmasiri, S., and Estelle, M. (2005). The F-box protein TIR1 is an auxin receptor. Nature. 435, 441-445. doi: 10.1038/nature03543

Ding, Z., Galvan-Ampudia, C. S., Demarsy, E., Langowski, L., Kleine-Vehn, J., Fan, Y., et al. (2011). Light-mediated polarization of the PIN3 auxin transporter for the phototropic response in Arabidopsis. Nat. Cell. Biol. 13, 447-452. doi: $10.1038 /$ ncb2208

Du, Q., and Kende, H. (2001). Expression of two HOOKLESS genes in peas (Pisum sativum L.). Plant Cell Physiol. 42, 374-378. doi: 10.1093/pcp/pce044

Fairchild, C. D., Schumaker, M. A., and Quail, P. H. (2000). HFR1 encodes an atypical bHLH protein that acts in phytochrome A signal transduction. Genes Dev. 14, 2377-2391. doi: 10.1101/gad.828000
Feng, S., Martinez, C., Gusmaroli, G., Wang, Y., Zhou, J., Wang, F., et al. (2008). Coordinated regulation of Arabidopsis thaliana development by light and gibberellins. Nature 451, 475-479. doi: 10.1038/nature06448

Folta, K. M., Pontin, M. A., Karlin-Neumann, G., Bottini, R., and Spalding, E. P. (2003). Genomic and physiological studies of early cryptochrome 1 action demonstrate roles for auxin and gibberellin in the control of hypocotyl growth by blue light. Plant J. 36, 203-214. doi: 10.1046/j.1365-313X.2003.01870.x

Foo, E., Ross, J. J., Davies, N. W., Reid, J. B., and Weller, J. L. (2006). A role for ethylene in the phytochrome-mediated control of vegetative development. Plant J. 46, 911-921. doi: 10.1111/j.1365-313X.2006.02754.x

Fox, A., Soto, G., Jones, A., Casal, J., Muschietti, J., and Mazzella, M. (2012). cryl and GPAl signaling genetically interact in hook opening and anthocyanin synthesis in Arabidopsis. Plant Mol Biol. 80, 315-324. doi: 10.1007/s11103-0129950-x

Gallego-Bartolomé, J., Arana, M. V., Vandenbussche, F., Zadnikova, P., Minguet, E. G., Guardiola, V., et al. (2011). Hierarchy of hormone action controlling apical hook development in Arabidopsis. Plant J. 67, 622-634. doi: 10.1111/j.1365313X.2011.04621.x

Gallego-Bartolome, J., Minguet, E. G., Grau-Enguix, F., Abbas, M., Locascio, A., Thomas, S. G., et al. (2012). Molecular mechanism for the interaction between gibberellin and brassinosteroid signaling pathways in Arabidopsis. Proc. Natl. Acad. Sci. U.S.A. 109, 13446-13451. doi: 10.1073/pnas.1119992109

Gendron, J. M., Haque, A., Gendron, N., Chang, T., Asami, T., and Wang, Z. Y. (2008). Chemical genetic dissection of brassinosteroid-ethylene interaction. Mol. Plant. 1, 368-379. doi: 10.1093/mp/ssn005

Guzman, P., and Ecker, J. R. (1990). Exploiting the triple response of Arabidopsis to identify ethylene-related mutants. Plant Cell 2, 513-523. doi: 10.1105/tpc.2.6.513

Harper, R. M., Stowe-Evans, E. L., Luesse, D. R., Muto, H., Tatematsu, K., Watahiki, M. K., et al. (2000). The NPH4 locus encodes the auxin response factor ARF7, a conditional regulator of differential growth in aerial Arabidopsis tissue. Plant Cell 12, 757-770. doi: 10.2307/3870999

Hayashi, K. (2012). The interaction and integration of auxin signaling components. Plant Cell Physiol. 53, 965-975. doi: 10.1093/pcp/pcs035

Hiltbrunner, A., Tscheuschler, A., Viczian, A., Kunkel, T., Kircher, S., and Schafer, E. (2006). FHY1 and FHL act together to mediate nuclear accumulation of the phytochrome A photoreceptor. Plant Cell Physiol. 47, 1023-1034. doi: $10.1093 / \mathrm{pcp} / \mathrm{pcj} 087$

Hirose, F., Inagaki, N., Hanada, A., Yamaguchi, S., Kamiya, Y., Miyao, A., et al. (2012). Cryptochrome and phytochrome cooperatively but independently reduce active gibberellin content in rice seedlings under light irradiation. Plant Cell Physiol. 53, 1570-1582. doi: 10.1093/pcp/pcs097

Hisada, A., Hanzawa, H., Weller, J. L., Nagatani, A., Reid, J. B., and Furuya, M. (2000). Light-induced nuclear translocation of endogenous pea phytochrome A visualized by immunocytochemical procedures. Plant Cell 12, 1063-1078. doi: $10.2307 / 3871255$

Hua, J., and Meyerowitz, E. M. (1998). Ethylene responses are negatively regulated by a receptor gene family in Arabidopsis thaliana. Cell. 94, 261-271. doi: 10.1016/S0092-8674(00)81425-7

Hua, J., Sakai, H., Nourizadeh, S., Chen, Q. G., Bleecker, A. B., Ecker, J. R., et al. (1998). EIN4 and ERS2 are members of the putative ethylene receptor gene family in Arabidopsis. Plant Cell 10, 1321-1332. doi: 10.2307/3870643

Huq, E., Al-Sady, B., and Quail, P. H. (2003). Nuclear translocation of the photoreceptor phytochrome B is necessary for its biological function in seedling photomorphogenesis. Plant J. 35, 660-664. doi: 10.1046/j.1365-313X.2003.01836.x

Kami, C., Lorrain, S., Hornitschek, P., and Fankhauser, C. (2010). Chapter two light-regulated plant growth and development. Curr. Top. Dev. Biol. 91, 29-66. doi: 10.1016/S0070-2153(10)91002-8

Khanna, R., Shen, Y., Marion, C. M., Tsuchisaka, A., Theologis, A., Schafer, E., et al. (2007). The basic helix-loop-helix transcription factor PIF5 acts on ethylene biosynthesis and phytochrome signaling by distinct mechanisms. Plant Cell 19, 3915-3929. doi: 10.1105/tpc.107.051508

Kim, K., Shin, J., Lee, S.-H., Kweon, H.-S., Maloof, J. N., and Choi, G. (2011). Phytochromes inhibit hypocotyl negative gravitropism by regulating the development of endodermal amyloplasts through phytochrome-interacting factors. Proc. Natl. Acad. Sci. U.S.A. 108, 1729-1734. doi: 10.1073/pnas.1011066108

Kircher, S., Gil, P., Kozma-Bognar, L., Fejes, E., Speth, V., Husselstein-Muller, T., et al. (2002). Nucleocytoplasmic partitioning of the plant photoreceptors 
phytochrome A, B, C, D, and E is regulated differentially by light and exhibits a diurnal rhythm. Plant Cell 14, 1541-1555. doi: 10.1105/tpc.001156

Kircher, S., Wellmer, F., Nick, P., Rugner, A., Schafer, E., and Harter, K. (1999). Nuclear import of the parsley bZIP transcription factor CPRF2 is regulated by phytochrome photoreceptors. J. Cell Biol. 144, 201-211. doi: 10.1083/jcb.144.2.201

Knee, E. M., Hangarter, R. P., and Knee, M. (2000). Interactions of light and ethylene in hypocotyl hook maintenance in Arabidopsis thaliana seedlings. Physiol Plant. 108, 208-215. doi: 10.1034/j.1399-3054.2000.108002208.x

Kutschera, U., and Niklas, K. J. (2013). Cell division and turgor-driven stem elongation in juvenile plants: a synthesis. Plant Sci. 207, 45-56. doi: 10.1016/j.plantsci.2013.02.004

Lau, O. S., and Deng, X. W. (2012). The photomorphogenic repressors COP1 and DET1: 20 years later. Trends Plant Sci. 17, 584-593. doi: 10.1016/j.tplants.2012.05.004

Lee, J., He, K., Stolc, V., Lee, H., Figueroa, P., Gao, Y., et al. (2007). Analysis of transcription factor HY5 genomic binding sites revealed its hierarchical role in light regulation of development. Plant Cell 19, 731-749. doi: 10.1105/tpc.106. 047688

Lehman, A., Black, R., and Ecker, J. R. (1996). HOOKLESS1, an ethylene response gene, is required for differential cell elongation in the Arabidopsis hypocotyl. Cell 85, 183-194. doi: 10.1016/S0092-8674(00)81095-8

Leivar, P., Monte, E., Oka, Y., Liu, T., Carle, C., Castillon, A., et al. (2008). Multiple phytochrome-interacting bHLH transcription factors repress premature seedling photomorphogenesis in darkness. Curr Biol. 18, 1815-1823. doi: 10.1016/j.cub.2008.10.058

Leivar, P., and Quail, P. H. (2011). PIFs: pivotal components in a cellular signaling hub. Trends Plant Sci. 16, 19-28. doi: 10.1016/j.tplants.2010.08.003

Leivar, P., Tepperman, J. M., Monte, E., Calderon, R. H., Liu, T. L., and Quail, P. H. (2009). Definition of early transcriptional circuitry involved in lightinduced reversal of PIF-imposed repression of photomorphogenesis in young Arabidopsis seedlings. Plant Cell 21, 3535-3553. doi: 10.1105/tpc.109.070672

Li, H., Johnson, P., Stepanova, A., Alonso, J. M., and Ecker, J. R. (2004). Convergence of signaling pathways in the control of differential cell growth in Arabidopsis. Dev. Cell. 7, 193-204. doi: 10.1016/j.devcel.2004.07.002

Li, J., Nagpal, P., Vitart, V., McMorris, T. C., and Chory, J. (1996). A role for brassinosteroids in light-dependent development of Arabidopsis. Science. 272, 398-401. doi: 10.1126/science.272.5260.398

Li, Y., Swaminathan, K., and Hudson, M. E. (2011). Rapid, organ-specific transcriptional responses to light regulate photomorphogenic development in dicot seedlings. Plant Physiol. 156, 2124-2140. doi: 10.1104/pp.111.179416

Lian, H. L., He, S. B., Zhang, Y. C., Zhu, D. M., Zhang, J. Y., Jia, K. P., et al. (2011). Blue-light-dependent interaction of cryptochrome 1 with SPA1 defines a dynamic signaling mechanism. Genes Dev. 25, 1023-1028. doi: 10.1101/gad. 2025111

Liang, X., Wang, H., Mao, L., Hu, Y., Dong, T., Zhang, Y., et al. (2012). Involvement of COP1 in ethylene- and light-regulated hypocotyl elongation. Planta. 236, 1791-1802. doi: 10.1007/s00425-012-1730-y

Liscum, E., and Hangarter, R. P. (1993a). Light-stimulated apical hook opening in wild-type Arabidopsis thaliana seedlings. Plant Physiol. 101, 567-572.

Liscum, E., and Hangarter, R. P. (1993b). Photomorphogenic mutants of Arabidopsis thaliana reveal activities of multiple photosensory systems during light-stimulated apical-hook opening. Planta 191, 214-221. doi: 10.1007/BF00199752

Liu, B., Zuo, Z., Liu, H., Liu, X., and Lin, C. (2011). Arabidopsis cryptochrome 1 interacts with SPA1 to suppress COP1 activity in response to blue light. Genes Dev. 25, 1029-1034. doi: 10.1101/gad.2025011

Liu, Q., and Wen, C. K. (2012). Arabidopsis ETR1 and ERS1 differentially repress the ethylene response in combination with other ethylene receptor genes. Plant Physiol. 158, 1193-1207. doi: 10.1104/pp.111.187757

Mazzella, M. A., Arana, M. V., Staneloni, R. J., Perelman, S., Rodriguez Batiller, M. J., Muschietti, J., et al. (2005). Phytochrome control of the Arabidopsis transcriptome anticipates seedling exposure to light. Plant Cell 17, 2507-2516. doi: 10.1105/tpc.105.034322

Nagashima, A., Suzuki, G., Uehara, Y., Saji, K., Furukawa, T., Koshiba, T., et al. (2008). Phytochromes and cryptochromes regulate the differential growth of Arabidopsis hypocotyls in both a PGP19-dependent and a PGP19independent manner. Plant J. 53, 516-529. doi: 10.1111/j.1365-313X.2007. 03358.x
Neff, M. M., and Chory, J. (1998). Genetic interactions between phytochrome A, phytochrome B, and cryptochrome 1 during Arabidopsis development. Plant Physiol. 118, 27-35. doi: 10.1104/pp.118.1.27

O’Neill, D. P., Ross, J. J., and Reid, J. B. (2000). Changes in gibberellin A(1) levels and response during de-etiolation of pea seedlings. Plant Physiol. 124, 805-812. doi: 10.1104/pp.124.2.805

Osterlund, M. T., Ang, L. H., and Deng, X. W. (1999). The role of COP1 in repression of Arabidopsis photomorphogenic development. Trends Cell Biol. 9, 113-118. doi: 10.1016/S0962-8924(99)01499-3

Osterlund, M. T., Hardtke, C. S., Wei, N., and Deng, X. W. (2000). Targeted destabilization of HY5 during light-regulated development of Arabidopsis. Nature. 405, 462-466. doi: 10.1038/35013076

Pacín, M., Legris, M., and Casal, J. J. (2014). Rapid decline in nuclear COP1 abundance anticipates the stabilisation of its target HY5 in the light. Plant Physiol. doi: 10.1104/pp.113.234245. [Epub ahead of print].

Park, E., Park, J., Kim, J., Nagatani, A., Lagarias, J. C., and Choi, G. (2012). Phytochrome B inhibits binding of Phytochrome-Interacting Factors to their target promoters. Plant J. 72, 537-546. doi: 10.1111/j.1365-313X.2012.05114.x

Park, J., Kim, Y., Yoon, H., and Park, C. (2007). Functional characterization of a small auxin-up RNA gene in apical hook development in Arabidopsis. Plant Science. 172, 150-157. doi: 10.1016/j.plantsci.2006.08.005

Peck, S. C., Pawlowski, K., and Kende, H. (1998). Asymmetric responsiveness to ethylene mediates cell elongation in the apical hook of peas. Plant Cell 10, 713-720. doi: 10.2307/3870659

Peng, J., Carol, P., Richards, D. E., King, K. E., Cowling, R. J., Murphy, G. P., et al. (1997). The Arabidopsis GAI gene defines a signaling pathway that negatively regulates gibberellin responses. Genes Develop. 11, 3194-3205. doi: 10.1101/gad.11.23.3194

Powell, R. D., and Morgan, P. W. (1970). Factors involved in the opening of the hypocotyl hook of cotton and beans. Plant Physiol. 45, 548-552. doi: 10.1104/pp.45.5.548

Qu, X., Hall, B. P., Gao, Z., and Schaller, G. E. (2007). A strong constitutive ethylene-response phenotype conferred on Arabidopsis plants containing null mutations in the ethylene receptors ETR1 and ERS1. BMC Plant Biol. 7:3. doi: 10.1186/1471-2229-7-3

Quail, P. H., Boylan, M. T., Parks, B. M., Short, T. W., Xu, Y., and Wagner, D. (1995). Phytochromes: photosensory perception and signal transduction. Science. 268, 675-680. doi: 10.1126/science.7732376

Rausenberger, J., Tscheuschler, A., Nordmeier, W., Wust, F., Timmer, J., Schafer, E., et al. (2011). Photoconversion and nuclear trafficking cycles determine phytochrome A's response profile to far-red light. Cell 146, 813-825. doi: 10.1016/j.cell.2011.07.023

Raz, V., and Ecker, J. R. (1999). Regulation of differential growth in the apical hook of Arabidopsis. Development. 126, 3661-3668.

Raz, V., and Koornneef, M. (2001). Cell division activity during apical hook development. Plant Physiol. 125, 219-226. doi: 10.1104/pp.125.1.219

Reed, J. W., Nagatani, A., Elich, T. D., Fagan, M., and Chory, J. (1994). Phytochrome A and phytochrome B have overlapping but distinct functions in Arabidopsis development. Plant Physiol. 104, 1139-1149.

Reid, J. B., Botwright, N. A., Smith, J. J., O’Neill, D. P., and Kerckhoffs, L. H. (2002). Control of gibberellin levels and gene expression during de-etiolation in pea. Plant Physiol. 128, 734-741. doi: 10.1104/pp.010607

Rosler, J., Klein, I., and Zeidler, M. (2007). Arabidopsis fhl/fhyl double mutant reveals a distinct cytoplasmic action of phytochrome A. Proc. Natl. Acad. Sci. U.S.A. 104, 10737-10742. doi: 10.1073/pnas.0703855104

Sakai, H., Hua, J., Chen, Q. G., Chang, C., Medrano, L. J., Bleecker, A. B., et al. (1998). ETR2 is an ETR1-like gene involved in ethylene signaling in Arabidopsis. Proc. Natl. Acad. Sci. U.S.A. 95, 5812-5817. doi: 10.1073/pnas.95.10.5812

Sassi, M., Lu, Y., Zhang, Y., Wang, J., Dhonukshe, P., Blilou, I., et al. (2012). COP1 mediates the coordination of root and shoot growth by light through modulation of PIN1- and PIN2-dependent auxin transport in Arabidopsis. Development 139, 3402-3412. doi: 10.1242/dev.078212

Schaller, G. E., and Kieber, J. J. (2002). Ethylene. Arabidopsis Book 1, e0071. doi: 10.1199/tab.0071

Sellaro, R., Crepy, M., Trupkin, S. A., Karayekov, E., Buchovsky, A. S., Rossi, C., et al. (2010). Cryptochrome as a sensor of the blue/green ratio of natural radiation in Arabidopsis. Plant Physiol. 154, 401-409. doi: 10.1104/pp.110.160820

Sentandreu, M., Martin, G., Gonzalez-Schain, N., Leivar, P., Soy, J., Tepperman, J. M., et al. (2011). Functional profiling identifies genes involved in organ-specific 
branches of the PIF3 regulatory network in Arabidopsis. Plant Cell 23, 3974-3991. doi: 10.1105/tpc.111.088161

Shen, Y., Khanna, R., Carle, C. M., and Quail, P. H. (2007). Phytochrome induces rapid PIF5 phosphorylation and degradation in response to red-light activation. Plant Physiol. 145, 1043-1051. doi: 10.1104/pp.107.105601

Shichijo, C., Ohuchi, H., Iwata, N., Nagatoshi, Y., Takahashi, M., Nakatani, E., et al. (2010). Light exaggerates apical hook curvature through phytochrome actions in tomato seedlings. Planta 231, 665-675. doi: 10.1007/s00425-009$1065-5$

Shin, J., Kim, K., Kang, H., Zulfugarov, I. S., Bae, G., Lee, C.-H., et al. (2009). Phytochromes promote seedling light responses by inhibiting four negativelyacting phytochrome-interacting factors. Proc. Natl. Acad. Sci. U.S.A. 106, 7660-7665. doi: 10.1073/pnas.0812219106

Shinomura, T., Nagatani, A., Hanzawa, H., Kubota, M., Watanabe, M., and Furuya, M. (1996). Action spectra for phytochrome A- and B-specific photoinduction of seed germination in Arabidopsis thaliana. Proc. Natl. Acad. Sci. U.S.A. 93, 8129-8133. doi: 10.1073/pnas.93.15.8129

Silverstone, A. L., Ciampaglio, C. N., and Sun, T.-P. (1998). The Arabidopsis RGA gene encodes a transcriptional regulator repressing the Gibberellin signal transduction pathway. Plant Cell 10, 155-169. doi: 10.2307/3870695

Spartz, A. K., Lee, S. H., Wenger, J. P., Gonzalez, N., Itoh, H., Inze, D., et al. (2012). The SAUR19 subfamily of SMALL AUXIN UP RNA genes promote cell expansion. Plant J. 70, 978-990. doi: 10.1111/j.1365-313X.2012.04946.x

Stepanova, A. N., Hoyt, J. M., Hamilton, A. A., and Alonso, J. M. (2005). A Link between ethylene and auxin uncovered by the characterization of two rootspecific ethylene-insensitive mutants in Arabidopsis. Plant Cell 17, 2230-2242. doi: $10.1105 /$ tpc. 105.033365

Stepanova, A. N., Robertson-Hoyt, J., Yun, J., Benavente, L. M., Xie, D. Y., Dolezal, K., et al. (2008). TAA1-mediated auxin biosynthesis is essential for hormone crosstalk and plant development. Cell 133, 177-191. doi: 10.1016/j.cell.2008.01.047

Tanaka, N., Itoh, H., Sentoku, N., Kojima, M., Sakakibara, H., Izawa, T., et al. (2011). The COP1 ortholog PPS regulates the juvenile-adult and vegetative-reproductive phase changes in rice. Plant Cell 23, 2143-2154. doi: $10.1105 /$ tpc.111.083436

Tatematsu, K., Kumagai, S., Muto, H., Sato, A., Watahiki, M. K., Harper, R. M., et al. (2004). MASSUGU2 encodes Aux/IAA19, an auxin-regulated protein that functions together with the transcriptional activator NPH4/ARF7 to regulate differential growth responses of hypocotyl and formation of lateral roots in Arabidopsis thaliana. Plant Cell 16, 379-393. doi: 10.1105/tpc. 018630

Tian, Q., and Reed, J. W. (1999). Control of auxin-regulated root development by the Arabidopsis thaliana SHY2/IAA3 gene. Development 126, 711-721.

Tiwari, S. B., Hagen, G., and Guilfoyle, T. (2003). The roles of auxin response factor domains in auxin-responsive transcription. Plant Cell 15, 533-543. doi: $10.1105 /$ tpc. 008417

Ueguchi-Tanaka, M., Nakajima, M., Motoyuki, A., and Matsuoka, M. (2007). Gibberellin receptor and its role in gibberellin signaling in plants. Annu. Rev. Plant Biol. 58, 183-198. doi: 10.1146/annurev.arplant.58.032806.103830

Ulmasov, T., Hagen, G., and Guilfoyle, T. J. (1999). Activation and repression of transcription by auxin-response factors. Proc. Natl. Acad. Sci. U.S.A. 96, 5844-5849. doi: 10.1073/pnas.96.10.5844

Ulmasov, T., Murfett, J.,Hagen, G., and Guilfoyle, T. J. (1997). Aux/IAA proteins repress expression of reporter genes containing natural and highly active synthetic auxin response elements. Plant Cell 9, 1963-1971. doi: 10.2307/ 3870557

Vandenbussche, F., Petrasek, J., Zadnikova, P., Hoyerova, K., Pesek, B., Raz, V., et al. (2010). The auxin influx carriers AUX1 and LAX3 are involved in auxinethylene interactions during apical hook development in Arabidopsis thaliana seedlings. Development 137, 597-606. doi: 10.1242/dev.040790

Vandenbussche, F., and Van Der Straeten, D. (2004). Shaping the shoot: a circuitry that integrates multiple signals. Trends Plant Sci. 9, 499-506. doi: 10.1016/j.tplants.2004.08.002

Vernoux, T., Brunoud, G., Farcot, E., Morin, V., Van den Daele, H., Legrand, J., et al. (2011). The auxin signalling network translates dynamic input into robust patterning at the shoot apex. Mol. Syst. Biol. 7, 508. doi: 10.1038/msb.2011.39

Vert, G., Walcher, C. L., Chory, J., and Nemhauser, J. L. (2008). Integration of auxin and brassinosteroid pathways by Auxin Response Factor 2. Proc. Natl. Acad. Sci. U.S.A. 105, 9829-9834. doi: 10.1073/pnas.0803996105
Vogel, J. P., Schuerman, P., Woeste, K., Brandstatter, I., and Kieber, J. J. (1998a). Isolation and characterization of Arabidopsis mutants defective in the induction of ethylene biosynthesis by cytokinin. Genetics 149, 417-427.

Vogel, J. P., Woeste, K. E., Theologis, A., and Kieber, J. J. (1998b). Recessive and dominant mutations in the ethylene biosynthetic gene ACS5 of Arabidopsis confer cytokinin insensitivity and ethylene overproduction, respectively. Proc. Natl. Acad. Sci. U.S.A. 95, 4766-4771. doi: 10.1073/pnas.95. 8.4766

von Arnim, A. G., and Deng, X. W. (1994). Light inactivation of Arabidopsis photomorphogenic repressor COP1 involves a cell-specific regulation of its nucleocytoplasmic partitioning. Cell 79, 1035-1045. doi: 10.1016/0092-8674(94) 90034-5

Vriezen, W. H., Achard, P., Harberd, N. P., and Van Der Straeten, D. (2004). Ethylene-mediated enhancement of apical hook formation in etiolated Arabidopsis thaliana seedlings is gibberellin dependent. Plant J. 37, 505-516. doi: 10.1046/j.1365-313X.2003.01975.X

Wang, H., Ma, L. G., Li, J. M., Zhao, H. Y., and Deng, X. W. (2001). Direct interaction of Arabidopsis cryptochromes with COP1 in light control development. Science 294, 154-158. doi: 10.1126/science. 1063630

Wang, L., Uilecan, I. V., Assadi, A. H., Kozmik, C. A., and Spalding, E. P. (2009). HYPOTrace: image analysis software for measuring hypocotyl growth and shape demonstrated on Arabidopsis seedlings undergoing photomorphogenesis. Plant Physiol. 149, 1632-1637. doi: 10.1104/pp.108. 134072

Weller, J. L., Hecht, V. R., Vander Schoor, J. K., Davidson, S. E., and Ross, J. J. (2009). Light Regulation of Gibberellin Biosynthesis in Pea Is Mediated through the COP1/HY5 Pathway. Plant Cell 21, 800-813. doi: 10.1105/tpc.108. 063628

Willige, B. C., Ghosh, S., Nill, C., Zourelidou, M., Dohmann, E. M., Maier, A., et al. (2007). The DELLA domain of GA INSENSITIVE mediates the interaction with the GA INSENSITIVE DWARF1A gibberellin receptor of Arabidopsis. Plant Cell 19, 1209-1220. doi: 10.1105/tpc.107.051441

Willige, B. C., Ogiso-Tanaka, E., Zourelidou, M., and Schwechheimer, C. (2012). WAG2 represses apical hook opening downstream from gibberellin and PHYTOCHROME INTERACTING FACTOR 5. Development 139, 4020-4028. doi: $10.1242 /$ dev. 081240

Woeste, K. E., Ye, C., and Kieber, J. J. (1999). Two Arabidopsis mutants that overproduce ethylene are affected in the posttranscriptional regulation of 1aminocyclopropane-1-carboxylic acid synthase. Plant Physiol. 119, 521-530. doi: 10.1104/pp.119.2.521

Wu, G., Cameron, J. N., Ljung, K., and Spalding, E. P. (2010). A role for ABCB19mediated polar auxin transport in seedling photomorphogenesis mediated by cryptochrome 1 and phytochrome B. Plant J. 62, 179-191. doi: 10.1111/j.1365313X.2010.04137.x

Wu, G., and Spalding, E. P. (2007). Separate functions for nuclear and cytoplasmic cryptochrome 1 during photomorphogenesis of Arabidopsis seedlings. Proc. Natl. Acad. Sci. U.S.A. 104, 18813-18818. doi: 10.1073/pnas.0705082104

Yang, X., Lee, S., So, J. H., Dharmasiri, S., Dharmasiri, N., Ge, L., et al. (2004). The IAA1 protein is encoded by AXR5 and is a substrate of SCF(TIR1). Plant J. 40, 772-782. doi: 10.1111/j.1365-313X.2004.02254.X

Yi, C., and Deng, X. W. (2005). COP1 - from plant photomorphogenesis to mammalian tumorigenesis. Trends Cell Biol. 15, 618-625. doi: 10.1016/j.tcb.2005.09.007

Zadnikova, P., Petrasek, J., Marhavy, P., Raz, V., Vandenbussche, F., Ding, Z., et al. (2010). Role of PIN-mediated auxin efflux in apical hook development of Arabidopsis thaliana. Development 137, 607-617. doi: 10.1242/dev. 041277

Zhang, H., He, H., Wang, X., Yang, X., Li, L., and Deng, X. W. (2013a). Genomewide mapping of the HY5-mediated gene networks in Arabidopsis that involve both transcriptional and post-transcriptional regulation. Plant J. 65, 346-358. doi: 10.1111/j.1365-313X.2010.04426.x

Zhang, Y., Mayba, O., Pfeiffer, A., Shi, H., Tepperman, J. M., Speed, T. P., et al. (2013b). A quartet of PIF bHLH factors provides a transcriptionally centered signaling hub that regulates seedling morphogenesis through differential expression-patterning of shared target genes in Arabidopsis. PLoS Genet. 9:e1003244. doi: 10.1371/journal.pgen.1003244

Zhao, X., Yu, X., Foo, E., Symons, G. M., Lopez, J., Bendehakkalu, K. T., et al. (2007). A study of gibberellin homeostasis and cryptochrome-mediated blue 
light inhibition of hypocotyl elongation. Plant Physiol. 145, 106-118. doi: 10.1104/pp.107.099838

Zhao, Y., Christensen, S. K., Fankhauser, C., Cashman, J. R., Cohen, J. D., Weigel, D., et al. (2001). A role for flavin monooxygenase-like enzymes in auxin biosynthesis. Science 291, 306-309. doi: 10.1126/science.291.5502.306

Zhong, S., Zhao, M., Shi, T., Shi, H., An, F., Zhao, Q., et al. (2009). EIN3/EIL1 cooperate with PIF1 to prevent photo-oxidation and to promote greening of Arabidopsis seedlings. Proc. Natl. Acad. Sci. U.S.A. 106, 21431-21436. doi: 10.1073/pnas.0907670106

Conflict of Interest Statement: The authors declare that the research was conducted in the absence of any commercial or financial relationships that could be construed as a potential conflict of interest.
Received: 26 September 2013; paper pending published: 28 October 2013; accepted: 04 February 2014; published online: 26 February 2014.

Citation: Mazzella MA, Casal JJ, Muschietti JP and Fox AR (2014) Hormonal networks involved in apical hook development in darkness and their response to light. Front. Plant Sci. 5:52. doi: 10.3389/fpls.2014.00052

This article was submitted to Plant Physiology, a section of the journal Frontiers in Plant Science.

Copyright $\odot 2014$ Mazzella, Casal, Muschietti and Fox. This is an open-access article distributed under the terms of the Creative Commons Attribution License (CC BY). The use, distribution or reproduction in other forums is permitted, provided the original author(s) or licensor are credited and that the original publication in this journal is cited, in accordance with accepted academic practice. No use, distribution or reproduction is permitted which does not comply with these terms. 